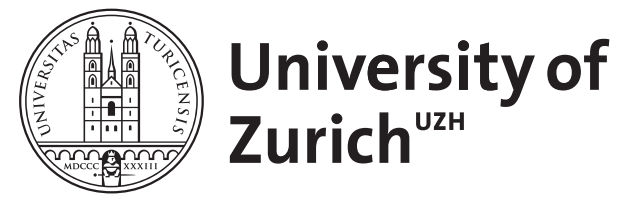

\title{
Asymmetric reduced form auctions
}

Mierendorff, Konrad

\begin{abstract}
This paper provides a tractable characterization of feasibility of asymmetric reduced form auctions. Using this, auction design problems can be stated in terms of the reduced form only. This allows to solve optimal auction problems when classical solution techniques fail.
\end{abstract}

DOI: https://doi.org/10.1016/j.econlet.2010.09.019

Posted at the Zurich Open Repository and Archive, University of Zurich ZORA URL: https://doi.org/10.5167/uzh-42849

Journal Article

Accepted Version

Originally published at:

Mierendorff, Konrad (2011). Asymmetric reduced form auctions. Economics Letters, 110(1):41-44.

DOI: https://doi.org/10.1016/j.econlet.2010.09.019 


\title{
Asymmetric Reduced Form Auctions ${ }^{\text {th }}$
}

\author{
Konrad Mierendorff* \\ Institute for Empirical Research in Economics, University of Zürich, Blümlisalpstrasse \\ 10, 8006 Zürich, Switzerland
}

\begin{abstract}
This paper provides a tractable characterization of feasibility of asymmetric reduced form auctions. Using this, auction design problems can be stated in terms of the reduced form only. This allows to solve optimal auction problems when classical solution techniques fail.
\end{abstract}

Keywords: Asymmetric Auction, Reduced Form, Feasibility JEL-Codes: D44

\section{Introduction}

The classical approach to solve optimal auction problems is a two-step procedure (Myerson, 1981). Bayesian incentive constraints are used to eliminate the payment rule from the designer's objective function. Then, the objective is maximized point-wise for each profile of types, subject to a feasibility or capacity constraint. This procedure avoids the problem that the feasibility constraint is formulated point-wise for each type-profile, whereas Bayesian incentive constraints are expressed in terms of the reduced form allocation rule. (The reduced form allocation rule is given by the interim winning probabilities of the bidders.) When the classical approach fails, a

\footnotetext{
This paper was written while I was visiting Paris School of Economics. I would like to thank PSE for its hospitality. Financial support of the Bonn Graduate School of Economics and the German Academic Exchange Service is gratefully acknowledged. I would like to thank Benny Moldovanu for his advice and Susanne Ohlendorf and Thomas Viehmann for helpful discussions.

*email-address: mierendorff@iew.uzh.ch, phone: +41 (0)44 63455 63, fax: +41 (0)44 6344907
} 
characterization of feasible reduced form allocation rules is needed to solve the optimization problem. ${ }^{1}$

Proving a conjecture of Matthews (1984), Border (1991) solved this question for the case of symmetric allocation rules. Symmetric reduced form allocation rules are feasible if and only if for each measurable subset $A$ of the type-space, the probability that the object is allocated to a bidder with type in $A$ does not exceed the probability that there is a bidder with type in $A$. Following Border (2007), we call this condition the Maskin-Riley-Mathews (MRM) condition. Border also shows that it is sufficient to check the MRM condition on a one-dimensional family of sets $A$ - the level sets of the reduced form allocation rule. This yields a tractable characterization for feasibility.

Border (2007) generalizes the first result to asymmetric environments with finite type spaces. In this paper, we identify a low dimensional family of sets of type profiles such that the MRM condition is sufficient for feasibility when applied to this family. The family is a suitable generalization of the family of level sets in Border (1991). In this way, we obtain a tractable characterization of feasibility in the asymmetric case with Border (1991) as a special case. ${ }^{2}$ The proof is based on Border (1991) but requires some non-trivial extensions for the asymmetric case.

\section{Definitions and Results}

There are $N$ bidders $i=1, \ldots, N$. Each bidder belongs to one of $L \leq N$ groups $l=1, \ldots, L . \gamma(i)$ denotes the group of bidder $i, \gamma^{-1}(l)$ denotes the set of bidders that belong to group $l$, and $N^{l}=\left|\gamma^{-1}(l)\right|$ denotes the number of bidders in group $l$. (The sets $\gamma^{-1}(1), \ldots, \gamma^{-1}(L)$ partition the set of bidders $\{1, \ldots, N\}$. Hence $\sum_{l=1}^{L} N^{l}=N$.) For each group $l$, there is a probability space $\left(T^{l}, \mathcal{T}^{l}, \mu^{l}\right)$. The type of a bidder $i$ is denoted $t^{i} \in T^{\gamma(i)}$. The types of all bidders that belong to group $l$ are identically distributed according to the probability measure $\mu^{l}$. The space of all type profiles $t=\left(t^{1}, \ldots, t^{N}\right)$ is the product space of all type-spaces and is denoted by $(T, \mathcal{T}, \mu)=\left(T^{\gamma(1)} \times\right.$ $\left.T^{\gamma(2)} \times \ldots \times T^{\gamma(N)}, \mathcal{T}^{\gamma(1)} \otimes \mathcal{T}^{\gamma(2)} \otimes \ldots \otimes \mathcal{T}^{\gamma(N)}, \mu=\prod_{i=1}^{N} \mu^{\gamma(i)}\right)$. As usual, a type profile where the type of bidder $i$ is excluded, is denoted by $t^{-i}$ with probability space $\left(T^{-i}, \mathcal{T}^{-i}, \mu^{-i}\right) . \quad \theta=\left(\theta^{1}, \ldots, \theta^{L}\right)$ denotes a profile of $L$

\footnotetext{
${ }^{1}$ Maskin and Riley (1984) seems to be the first paper that solves an optimal auction problem when the classical approach fails.

${ }^{2}$ See Mierendorff (2009) for an application.
} 
types, one for each group. The associated probability space is $(\hat{T}, \hat{\mathcal{T}}, \hat{\mu})=$ $\left(T^{1} \times \ldots \times T^{L}, \mathcal{T}^{1} \otimes \ldots \otimes \mathcal{T}^{L}, \prod_{l=1}^{L} \mu^{l}\right)$.

Definition 1. (a) An allocation rule is a measurable function $q: T \rightarrow$ $[0,1]^{N}$ that satisfies the feasibility condition:

$$
\forall t \in T: \quad \sum_{i=1}^{N} q^{i}(t) \leq 1 .
$$

(b) An allocation rule is group symmetric if for all $l \in\{1, \ldots, L\}, i, j \in$ $\gamma^{-1}(l)$ and $t \in T$

$$
q^{i}(t)=q^{j}\left(\sigma_{i, j}(t)\right),
$$

where $\sigma_{i, j}$ interchanges the $i^{\text {th }}$ with the $j^{\text {th }}$ component of its argument.

$q^{i}(t)$ is the probability that bidder $i$ gets the object if the profile of types is $t$. The set of all group symmetric allocation rules is denoted by $\mathcal{Q}_{0}$. The (group) reduced form $\hat{Q}: \hat{T} \rightarrow[0,1]^{L}$ of $q \in Q_{0}$ is given by the interim winning probabilities

$$
\hat{Q}^{l}(\theta):=\int_{T^{-i}} q^{i}\left(\theta^{l}, t^{-i}\right) d \mu^{-i}\left(t^{-i}\right), \quad \text { where } \gamma(i)=l .
$$

The $l^{\text {th }}$ component of $\hat{Q}(\theta)$ is the probability that a bidder from group $l$ gets the object when his type is $\theta^{l}$. For all $l, \hat{Q}^{l}$ does not depend on $\theta^{-l}$. In what follows, a function with this property shall be called diagonal. A measurable diagonal function $\hat{Q}: \hat{T} \rightarrow[0,1]^{L}$ is called feasible if it is the reduced form of a group symmetric allocation rule $q \in \mathcal{Q}_{0}$. The set of all such functions is denoted by $\hat{\mathcal{Q}}$.

Let $\mathcal{F}$ denote the set of $L$-tuples of measurable subsets of the individual type-spaces ${ }^{3}$

$$
\mathcal{F}:=\left\{\left(A^{1}, \ldots, A^{L}\right) \mid \forall l: A^{l} \in \mathcal{T}^{l}\right\} .
$$

Two elements $A, B \in \mathcal{F}$ are called $\mathcal{F}$-disjoint (denoted $A \cap_{\mathcal{F}} B=\emptyset$ ) if for all $l=1, \ldots, L: A^{l} \cap B^{l}=\emptyset$.

\footnotetext{
${ }^{3}$ For each $A \in \mathcal{F}, A^{1} \times \ldots \times A^{L}$ is a subset of $\hat{T}$. The converse, however, is not true. This is a difference to the approach taken by Border (2007) who considers general subsets of $\hat{T}$ (and assumes $N=L$ ).
} 
Theorem 2. Let $\hat{Q}: \hat{T} \rightarrow[0,1]^{L}$ be measurable and diagonal. Then $\hat{Q} \in \hat{\mathcal{Q}}$ if and only if for each $A \in \mathcal{F}$,

$$
\sum_{l=1}^{L} N^{l} \int_{A^{l}} \hat{Q}^{l}\left(t^{l}\right) d \mu^{l}\left(t^{l}\right) \leq 1-\prod_{l=1}^{L}\left(1-\mu^{l}\left(A^{l}\right)\right)^{N^{l}} .
$$

This generalizes Theorem 3.1 in Border (1991). As in the symmetric case, the family of sets $A \in \mathcal{F}$ for which (MRM) must be checked can be reduced. In the group symmetric case, the reduced family has dimension $L$.

Theorem 3. Let $\hat{Q}: \hat{T} \rightarrow[0,1]^{L}$ be measurable and diagonal. For each $\alpha \in[0,1]^{L}$ let $E_{\alpha}=\left(E_{\alpha}^{1}, \ldots, E_{\alpha}^{L}\right)$ be given by $E_{\alpha}^{l}:=\left\{t^{l} \in T^{l} \mid \hat{Q}^{l}\left(t^{l}\right) \geq \alpha^{l}\right\}$. Then $\hat{Q} \in \hat{\mathcal{Q}}$ if and only if for each $\alpha \in[0,1]^{L}$, (MRM) is satisfies for $A=E_{\alpha}$.

\section{Proofs}

For the proofs, we use the bidder reduced form $Q: T \rightarrow[0,1]^{N}$ of a feasible group-symmetric allocation rule $q$ is defined as

$$
Q^{i}\left(t^{i}\right):=\int_{T^{-i}} q^{i}\left(t^{i}, t^{-i}\right) d \mu^{-i}\left(t^{-i}\right) .
$$

The set of bidder reduced forms of feasible and group symmetric allocation rules is denoted by $\mathcal{Q}$. Each $Q \in \mathcal{Q}$ is called feasible. Each $Q \in \mathcal{Q}$ is diagonal and group-symmetric $\left(Q^{i}(t)=Q^{j}(t)\right.$ for all $t \in T$ if $\left.i \in \gamma(j)\right)$. Hence, each $Q \in \mathcal{Q}$ has a representation $\hat{Q} \in \hat{\mathcal{Q}}$ that satisfies $\hat{Q}^{l}=Q^{i}$ if $l=\gamma(i)$ and vice versa.

As in Border (1991), hierarchical allocation rules are an important tool in the proofs. This notion has to be generalized to fit the asymmetric case.

Definition 4. Let $A_{1}, \ldots, A_{K} \in \mathcal{F}$ be a family of pairwise $\mathcal{F}$-disjoint sets. The hierarchical allocation rule generated by $A_{1}, \ldots, A_{K}$, and denoted by $q_{A_{1}, \ldots, A_{K}}$, is defined as

$$
q_{A_{1}, \ldots, A_{K}}^{i}(t):= \begin{cases}\frac{1}{\left|\left\{j: t^{j} \in A_{k}^{\gamma(j)}\right\}\right|} & \text { if } t^{i} \in A_{k}^{\gamma(i)} \text { and } \nexists j: t^{j} \in A_{1}^{\gamma(j)} \cup \ldots \cup A_{k-1}^{\gamma(j)} \\ 0 & \text { otherwise. }\end{cases}
$$


$A_{1}, \ldots, A_{K}$ define a hierarchy of types. $A_{k}^{l}$ is the set of types of bidders in group $l$ that are at the $k$ th level of the hierarchy. $q_{A_{1}, \ldots, A_{K}}$ works as follows. If there are bidders with types at the first level, the object is given to one of these bidders with equal probability. Otherwise, the auctioneer checks whether there are bidders with types at the second level, and allocates the object with equal probability to one of them. The auctioneer continues until either he has allocated the object or until he has checked for bidders at all levels of the hierarchy. In the latter case the object is not sold.

\subsection{Proof of Theorem 2}

The general approach is the same as in Border (1991). Since $\mu$ is a finite measure, $\mathcal{Q}_{0}$ and $\mathcal{Q}$ are subsets of the Hilbert space $L_{2}\left(T, \mu, \mathbb{R}^{N}\right) .{ }^{4}$ For $Q, f \in$ $L_{2}\left(T, \mu, \mathbb{R}^{N}\right)$ the scalar product is denoted $\langle Q, f\rangle=\int_{T}(Q(t), f(t)) d \mu(t)$, where (., .) denotes the Euclidean scalar product in $\mathbb{R}^{N} . L_{2}\left(T, \mu, \mathbb{R}^{N}\right)$ will be abbreviated as $L_{2}^{N}$, and $L_{2}(T, \mu, \mathbb{R})$ as $L_{2}$.

We define the vector of indicator functions for $\left(A^{1}, \ldots, A^{L}\right)=A \in \mathcal{F}$, as $\chi_{A}(t):=\left(\chi_{A^{\gamma(1)}}\left(t^{1}\right), \ldots, \chi_{A^{\gamma(N)}}\left(t^{N}\right)\right)$ so that $\chi_{A}: T \rightarrow\{0,1\}^{N}$. Clearly, $\chi_{A} \in L_{2}^{N}$ if $A \in \mathcal{F}$. Furthermore, define $B(A):=1-\prod_{l=1}^{L}\left(1-\mu^{l}\left(A^{l}\right)\right)^{N^{l}}$. If $Q$ is diagonal, group symmetric, and a representation of $\hat{Q}$, then (MRM) can be rewritten as

$$
\left\langle Q, \chi_{A}\right\rangle \leq B(A) .
$$

With this notation, Lemmas 5.1 and 5.3 from Border (1991) can be reproduced for the asymmetric case.

Lemma 5 (cf. Lemma 5.1, Border (1991)). For all $A \in \mathcal{F}$ and all $Q \in \mathcal{Q}$,

$$
\left\langle Q, \chi_{A}\right\rangle \leq B(A) .
$$

Lemma 6 (cf. Lemma 5.3, Border (1991)). Let $Q: T \rightarrow[0,1]^{N}$ be measurable and suppose that the function $f=\sum_{j=1}^{M} \alpha_{j} \chi_{A_{j}}$ with $\alpha_{1}, \ldots, \alpha_{M} \in \mathbb{R}$ and $A_{1}, \ldots, A_{M} \in \mathcal{F}$ separates $Q$ from $\mathcal{Q}$. That is, for all $\tilde{Q} \in \mathcal{Q}$ :

$$
\left\langle Q, \sum_{j=1}^{M} \alpha_{j} \chi_{A_{j}}\right\rangle>\left\langle\tilde{Q}, \sum_{j=1}^{M} \alpha_{j} \chi_{A_{j}}\right\rangle .
$$

Then for some set $A \in \mathcal{F},\left\langle Q, \chi_{A}\right\rangle>B(A)$.

\footnotetext{
${ }^{4}$ Border (1991) uses $L_{\infty}$ and $L_{1}$ but of course his proofs are still valid if we use $L_{2}$.
} 
In Lemma 6, the simple function $f=\sum_{j=1}^{M} \alpha_{j} \chi_{A_{j}}$ is diagonal and group symmetric. The following Lemma implies that whenever a function $\tilde{f} \in L_{2}^{N}$ separates $Q$ from $\mathcal{Q}$, and $Q$ is diagonal and group symmetric, then there exists a diagonal group symmetric function $f \in L_{2}^{N}$ that separates $Q$ from $\mathcal{Q}$. Lemma 7 is not needed in the symmetric case.

Lemma 7. For every $\tilde{f} \in L_{2}^{N}$, there exist a diagonal and group symmetric $f \in L_{2}^{N}$ such that $\langle Q, \tilde{f}\rangle=\langle Q, f\rangle$ for all diagonal and group symmetric $Q \in L_{2}^{N}$.

Proof. Let $Q \in L_{2}^{N}$ be diagonal and group symmetric with representation $\hat{Q}: \hat{T} \rightarrow[0,1]^{L}$. Then,

$$
\begin{aligned}
\langle Q, \tilde{f}\rangle & =\int_{T} \sum_{i=1}^{N} \tilde{f}^{i}(t) Q^{i}(t) \mu(t), \\
& =\sum_{i=1}^{N} \int_{T^{i}}\left(\int_{T^{-i}} \tilde{f}^{i}\left(t^{i}, t^{-i}\right) Q^{i}\left(t^{i}, t^{-i}\right) d \mu^{-i}\left(t^{-i}\right)\right) d \mu^{\gamma(i)}\left(t^{i}\right), \\
& =\sum_{i=1}^{N} \int_{T^{i}} \underbrace{\left(\int_{T^{-i}} \tilde{f}^{i}\left(t^{i}, t^{-i}\right) d \mu^{-i}\left(t^{-i}\right)\right)}_{=\xi^{i}\left(t^{i}\right)} Q^{i}\left(t^{i}\right) d \mu^{\gamma(i)}\left(t^{i}\right), \\
& =\sum_{i=1}^{N} \int_{T^{i}} \xi^{i}\left(t^{i}\right) Q^{i}\left(t^{i}\right) d \mu^{\gamma(i)}\left(t^{i}\right), \\
& =\sum_{l=1}^{L} \sum_{i \in \gamma^{-1}(l)} \int_{T^{l}} \xi^{i}\left(t^{l}\right) \hat{Q}^{l}\left(t^{l}\right) d \mu^{l}\left(t^{l}\right), \\
& =\sum_{l=1}^{L} \int_{T^{l}} \underbrace{\left(\sum_{i \in \gamma^{-1}(l)} \xi^{i}\left(t^{l}\right)\right)}_{=: N^{l} \hat{f}^{l}\left(t^{l}\right)} \hat{Q}^{l}\left(t^{l}\right) d \mu^{l}\left(t^{l}\right), \\
& =\sum_{i=1}^{N} \int_{T^{i}} f^{i}\left(t^{i}\right) Q^{i}\left(t^{i}\right) d \mu^{\gamma(i)}\left(t^{i}\right), \\
& =\langle Q, f\rangle .
\end{aligned}
$$

$\xi: T \rightarrow[0,1]^{N}$ is diagonal by definition and therefore $\hat{f}: \Theta \rightarrow[0,1]^{L}$ is also diagonal. With $f: T \rightarrow[0,1]^{N}$ defined as $f^{i}(t)=\hat{f}^{\gamma(i)}\left(t^{i}\right)=$ 
$\frac{1}{N^{\gamma(i)}} \sum_{j: \gamma(j)=\gamma(i)} \xi^{j}\left(t^{i}\right)$, the desired diagonal and group symmetric function is obtained.

Lemma 8. $\mathcal{Q}_{0}$ and $\mathcal{Q}$ are weakly compact subsets of $L_{2}^{N}$.

Proof. The proof is very similar to the proof of Lemma 5.4 in Border (1991). Since we work with the Hilbert-Space $L_{2}^{N}$ and $\mathcal{Q}_{0}$ is bounded, we have that every sequence $\left(q_{n}\right)$ in $\mathcal{Q}_{0}$ has a weakly convergent subsequence (with limit in $L_{2}^{N}$ ). Following Border, it can be shown that $\mathcal{Q}_{0}$ is weakly closed and hence weakly compact. Furthermore, the mapping $\Lambda$, that associates an allocation rule with its reduced form is weakly continuous. As $\mathcal{Q}$ is the image of a compact set under $\Lambda$, it is also weakly compact.

Proof of Theorem 2. Let $\hat{Q}: \hat{T} \rightarrow[0,1]^{L}$ be diagonal. Then it is the representation of a diagonal and group symmetric function $Q: T \rightarrow[0,1]^{N}$.

Lemma 5 shows that condition (MRM) is necessary for feasibility. Conversely suppose $Q \notin \mathcal{Q}$. $\mathcal{Q}$ is a convex and weakly compact subset of $L_{2}^{N}$. By a standard separation theorem, ${ }^{5}$ there exists a function $f \in L_{2}^{N}$ such that $\langle Q, f\rangle>\max \{\langle\tilde{Q}, f\rangle \mid \tilde{Q} \in \mathcal{Q}\}$. By Lemma 7, $f$ can be chosen to be diagonal and group symmetric. Furthermore, as the simple functions are dense in $L_{2}$, we can take each component $f^{i}$ to be a simple function. Hence $f$ satisfies the conditions of Lemma 6 and there exists $A \in F$ such that (MRM) is violated. It remains to be shown that for every $Q \in \mathcal{Q}$ there exists a $q^{*} \in \mathcal{Q}_{0}$ such that $\Lambda\left(q^{*}\right)(t)=Q(t)$ for every $t \in T$ (so far this has been shown for almost every $t \in T$ ). The proof can be found in Border (1991) and is omitted here.

\subsection{Proof of Theorem 3}

As in the symmetric case, the proof of Theorem 3 starts by showing the result for simple functions. For $A \in \mathcal{F}$ and $\chi_{A}: T \rightarrow[0,1]^{N}$ as above, let $\hat{\chi}_{A}: \hat{T} \rightarrow[0,1]^{L}$ denote the representation of $\chi_{A}$.

Lemma 9. Let $\hat{Q}: \hat{T} \rightarrow[0,1]^{L}$ be a diagonal simple function with $\hat{Q}=$ $\sum_{k=1}^{K} \alpha_{k} \hat{\chi}_{A_{k}}$ where $\alpha_{1}>\alpha_{2}>\ldots>\alpha_{K} \geq 0$, the $A_{k} \in \mathcal{F}$ are pairwise $\mathcal{F}$ disjoint and $A_{1}^{l} \cup \ldots \cup A_{K}^{l}=T^{l}$ for all $l$. For $l=1, \ldots, L$ and $k=1, \ldots, K$

\footnotetext{
${ }^{5}$ cf. Theorem 3.4 in Rudin (1973)
} 
set $E_{k}^{l}:=A_{1}^{l} \cup \ldots \cup A_{k}^{l}$ and set $E_{0}^{l}:=\emptyset$.

If for each $\left(k_{1}, \ldots, k_{L}\right) \in\{0,1, \ldots, K\}^{L}$ :

$$
\sum_{l=1}^{L} N^{l} \int_{E_{k_{l}}^{l}} \hat{Q}\left(t^{l}\right) d \mu^{l}\left(t^{l}\right) \leq B\left(E_{k_{1}}^{1}, \ldots, E_{k_{L}}^{L}\right),
$$

then $\hat{Q} \in \hat{\mathcal{Q}}$.

Proof. Define $f:[0,1]^{L} \rightarrow[0,1]$ as $f(x):=1-\prod_{m=1}^{L}\left(1-x^{m}\right)^{N^{m}}$. This implies

$$
\partial_{l}^{2} f(x)=-N^{l}\left(N^{l}-1\right)\left(1-x^{l}\right)^{N^{l}-2} \prod_{m \neq l}\left(1-x^{m}\right)^{N^{m}} \leq 0,
$$

$$
\text { and } B(A)=f\left(\mu^{1}\left(A^{1}\right), \ldots, \mu^{L}\left(A^{L}\right)\right) \text {, for } A \in \mathcal{F} \text {. }
$$

To simplify notation define $c_{k}^{l}:=\mu^{l}\left(E_{k}^{l}\right)$. In order to bound the left hand side of (MRM), define $g:[0,1]^{L} \rightarrow[0,1]$ as a continuous and piecewise linear function with $g(0)=0$. For $x \in\left(c_{k_{1}-1}^{1}, c_{k_{1}}^{1}\right) \times \ldots \times\left(c_{k_{L}-1}^{L}, c_{k_{L}}^{L}\right)$, let the gradient of $g$ be given by

$$
\nabla g(x)=\left(\begin{array}{c}
N^{1} \alpha_{k_{1}} \\
\vdots \\
N^{L} \alpha_{k_{L}}
\end{array}\right) .
$$

With this definition, $g(x) \leq f(x)$ on the grid of points $G_{0}:=\left\{\left(c_{k_{1}}^{1}, \ldots, c_{k_{L}}^{L}\right) \mid k_{i} \in\right.$ $\{0,1, \ldots, L\}\}$ :

$$
\begin{aligned}
\forall x \in G_{0}: \quad g(x) & =\sum_{l=1}^{L} N^{l} \sum_{k=1}^{k_{l}} \alpha_{k} \mu^{l}\left(A_{k}^{l}\right) \\
& =\sum_{l=1}^{L} N^{l} \int_{E_{k_{l}}^{l}} \hat{Q}^{l}\left(t^{l}\right) \mu^{l}\left(t^{l}\right) \\
& \leq f\left(\mu^{l}\left(E_{k_{1}}^{1}\right), \ldots, \mu^{L}\left(E_{k_{L}}^{L}\right)\right)=f(x) .
\end{aligned}
$$

The second equality follows from the definition of $\hat{Q}$ and the inequality follows from (1) and (3).

Now it is shown inductively, that $g(x) \leq f(x)$ on the sets

$$
G_{n}:=\left\{x \in[0,1]^{L}|L-n \leq|\left\{j \mid \exists k_{j}: x^{j}=c_{k_{j}}^{j}\right\} \mid\right\},
$$


for $n=1, \ldots, L{ }^{6}$ Observe that $G_{L}=[0,1]^{L}$. Suppose that $g(x) \leq f(x)$ for all $x \in G_{n-1}$. Let $x \in G_{n}$. Then there exist $l$ and $k_{l}$ such that $\underline{x}=$ $\left(x^{1}, \ldots, x^{l-1}, c_{k_{l}-1}^{l}, x^{l+1}, \ldots, x^{L}\right)$ and $\bar{x}=\left(x^{1}, \ldots, x^{l-1}, c_{k_{l}}^{l}, x^{l+1}, \ldots, x^{L}\right)$ are in $G_{n-1}$ and $x=x(\delta)=(1-\delta) \underline{x}+\delta \bar{x}$ for some $\delta \in[0,1]$. As $\underline{x}$ and $\bar{x}$ differ only in the $l$ th coordinate, $f(x(\delta))$ is weakly concave as a function of $\delta$ by (2). Furthermore, as the gradient of $g$ is constant on sets of the form $\left(c_{k_{1}-1}^{1}, c_{k_{1}}^{1}\right) \times \ldots \times\left(c_{k_{L}-1}^{L}, c_{k_{L}}^{L}\right), g(x(\delta))$ is linear as a function of $\delta$. By the induction hypothesis, $g(\underline{x}) \leq f(\underline{x})$ and $g(\bar{x}) \leq f(\bar{x})$. Therefore also $g(x) \leq f(x)$.

Now, for $A \in \mathcal{F}$ define $h:\left[0, \mu^{1}\left(A^{1}\right)\right] \times \ldots \times\left[0, \mu^{L}\left(A^{L}\right)\right] \rightarrow[0,1]$ as a continuous and piecewise linear function with $h(0)=0$. For $x \in\left(\mu^{1}\left(A^{1} \cap\right.\right.$ $\left.\left.E_{k_{1}-1}^{1}\right), \mu^{1}\left(A^{1} \cap E_{k_{1}}^{1}\right)\right) \times \ldots \times\left(\mu^{L}\left(A^{L} \cap E_{k_{L}-1}^{L}\right), \mu^{L}\left(A^{L} \cap E_{k_{L}}^{L}\right)\right)$ let the gradient of $h$ be given by

$$
\nabla h(x)=\left(\begin{array}{c}
N^{1} \alpha_{k_{1}} \\
\vdots \\
N^{L} \alpha_{k_{L}}
\end{array}\right) .
$$

With this definition,

$$
h(A)=\sum_{l=1}^{L} N^{l} \int_{A^{l}} \hat{Q}^{l}\left(t^{l}\right) d \mu^{l}\left(t^{l}\right) .
$$

Furthermore for all $x$ and all $l: \nabla_{l} h(x) \leq \nabla_{l} g(x)$. Therefore $h(x) \leq g(x) \leq$ $f(x)$ which implies (MRM) for all sets $A \in \mathcal{F}$ and therefore $\hat{Q} \in \hat{\mathcal{Q}}$ by proposition 2 .

Proof of Theorem 3. The proof works as the proof of proposition 3.2 in Border (1991). For the asymmetric case $\hat{Q}$ is approximated by the sequence of simple functions $\hat{Q}_{n}: \hat{T} \rightarrow \mathbb{R}^{L}$ which is constructed such that $\hat{Q}_{n}^{l}(t)=\frac{k}{2^{n}}$ on $\left\{t \mid \frac{k}{2^{n}} \leq \hat{Q}^{l}(t)<\frac{k+1}{2^{n}}\right\}$.

\section{References}

Border, K. C., 1991. Implementation of reduced form auctions: A geometric approach. Econometrica 59 (4), 1175-1187.

\footnotetext{
${ }^{6}$ In the case $L=3$ and $K=1, G_{0}$ are the vertices of the cuboid $[0,1]^{3}, G_{1}$ are the edges, $G_{2}$ are the surfaces and $G_{3}$ is the cuboid itself.
} 
Border, K. C., 2007. Reduced form auctions revisited. Economic Theory 31, $167-181$.

Maskin, E., Riley, J., 1984. Optimal auctions with risk averse buyers. Econometrica 52 (6), 1473-1518.

Matthews, S. A., 1984. On the implementability of reduced form auctions. Econometrica 52 (6), 1519-1522.

Mierendorff, K., 2009. Optimal dynamic mechanism design with deadlines, university of Bonn, unpulished working paper.

Myerson, R. B., 1981. Optimal Auction Design. Mathematics of Operations Reseach 6, 58-63.

Rudin, W., 1973. Functional Analysis. McGraw-Hill, New York. 